\title{
LV-VE-3
}

\section{Tips for removing transjugular intrahepatic portosystemic shunt stent located deep to the union site of SMV \& splenic vein during liver transplantation}

\author{
Eui Soo HAN, Kwang-Woong LEE*
}

Department of Surgery, Seoul National University College of Medicine, Seoul, Korea

Introduction: Transjugular intrahepatic portosystemic shunt (TIPS) is usually used for patients with end-stage liver disease as a bridge to liver transplantation. Additional techniques are required for removing the stent to perform vessel anastomosis in liver transplantation.

Methods: A 57-year-old female had hepatitis B treated with Entecavir. She underwent TIPS 25 months before living donor transplantation. She had liver cirrhosis with splenomegaly and patent TIPS without bland thrombus in the portal vein on the CT scan.

Results: First, after performing full liver mobilization for total hepatectomy, we clamped the proximal hilum using a vessel clamp. And then, all hepatic veins were transected. Intrahepatic hilum transection was performed including intrahepatic portal vein. The TIPS stent's tip in the portal side was removed using the eversion technique. Since then, the TIPS stent's tip in the IVC side was removed after IVC total clamping. Case 2 had the stent's tip down to SMV, so the stent was cut at the middle after eversion as much as possible to anastomosis the portal vein.

Conclusions: Safe and accurate stent removal is required when performing liver transplantation in patients who have TIPS. 